\title{
AZIMUTHAL AND REGIONAL VARIATIONS OF CODA WAVES IN NEW ZEALAND
}

\author{
Michael J. Kozuch ${ }^{1}$ and Mark Chadwick ${ }^{1}$
}

\begin{abstract}
We present the results of using seismic waveform data to show that the elliptical nature of isoseismal distributions in New Zealand is related to regional structural trends. The data also suggest that there are regional and azimuthal variations in the attenuation of coda waves, which may need to be considered in ground motion attenuation relations.

We stacked over 20,000 waveforms from the New Zealand seismographic network. The data were filtered, normalized and stacked. Noisy or clipped records were down-weighted or removed. We also treated dense networks as a single station and generated a single stack for these networks. Stacks of shallow earthquake sources are presented by region and azimuth.

Variations in coda length throughout New Zealand suggest regions of high scattering. Strong azimuthal dependence in the coda is observed for non-volcanic zone stations. NE-SW waveform stacks, which follow the strike of the subduction zone, contain significantly longer codas than those with NW-SE raypaths. Long coda trains are also observed in the volcanic and geothermal zones yet there is little or no apparent azimuthal variation. These coda are particularly strong throughout the records which explains the difficulty analysts have had in picking $\mathrm{S}$ waves.
\end{abstract}

\section{INTRODUCTION}

A common feature to New Zealand isoseismal distributions is a dominant elliptical pattern oriented roughly NE-SW as illustrated in Figure 1 by Downes (1995). Prior work has also illustrated these strong ellipticities in most of the country with the exception of several volcanic zones and basins (Kozuch and Chadwick, 1997; Kozuch et al., 1996; VereJones et al., 1995; Smith, 1978, 1995). Some of these shapes were believed to be sampling artifacts due to population distributions or proximity to coastlines, yet Dowrick (1991) claims to find no systematic pattern to their shapes. The asymmetric shapes might also be due to fault rupture directivity, topography and site effects. However, the strong NE-SW trend of these ellipticities suggests that the elongation along strike with the subduction zone or structural trends might not just be a sampling artifact.

Modeling these isoseismals has often been difficult, particularly given the nature of the data which heavily relies on the distribution of felt reports and the dimensions of New Zealand. In general, the dimensions of isoseismals is dependent on magnitude $(\mathrm{m})$ and epicentral distance $(\mathrm{r})$ :

$$
\mathbf{I}=\mathbf{I o}(\mathrm{m}, \mathbf{r})
$$

but these shapes also vary with location throughout New Zealand. To show this one would then expand equation (1) to include a site dependence term (site = latitude/longitude) which allows the aspect ratio $(a / b)$ of the ellipses to vary throughout the country as well as the orientation of the ellipse (theta). The final distribution would then be best expressed as:

$$
\mathrm{I}=\mathrm{Io}(\mathrm{m}, \mathrm{r}, \text { site }, \mathrm{a} / \mathrm{b} \text {, theta })
$$

As part of a national attenuation study we set forth to verify the results of the New Zealand isoseismal work by searching for this variation in attenuation in waveform data. In this paper we show that the NE trending ellipticity of isoseismals is most likely caused by an azimuthal variation in attenuation which is observed in the coda of stacked waveforms.

\section{DEFINITION OF CODA WAVES}

The decaying wave train following $\mathrm{P}$ - or S-waves in a seismogram is known as coda. These waves have been shown to result from the backscattering of seismic waves by velocity and structural inhomogeneous conditions in the earth (Aki, 1969; Aki and Chouet, 1975). The scatterers may be cracks, faults, density or velocity anomalies and depending upon their nature and distribution, lead to varying degrees of attenuation. The attenuation distance is the average distance the seismic wave travels before being attenuated by $1 / \mathrm{e}$ (Pulli, 1984). This exponential damping of primary waves (either Por S-waves) into scattered coda waves is primarily a redistribution of the energy density (Sato, 1977; Pulli, 1984). A drop in the overall energy density, on the other hand, is a sign of absorption in the medium. Each of these is a form of attenuation and they are often called either scattering or intrinsic attenuation, respectively.

\footnotetext{
${ }^{1}$ Institute of Geological \& Nuclear Sciences, Limited, Lower Hutt.
} 


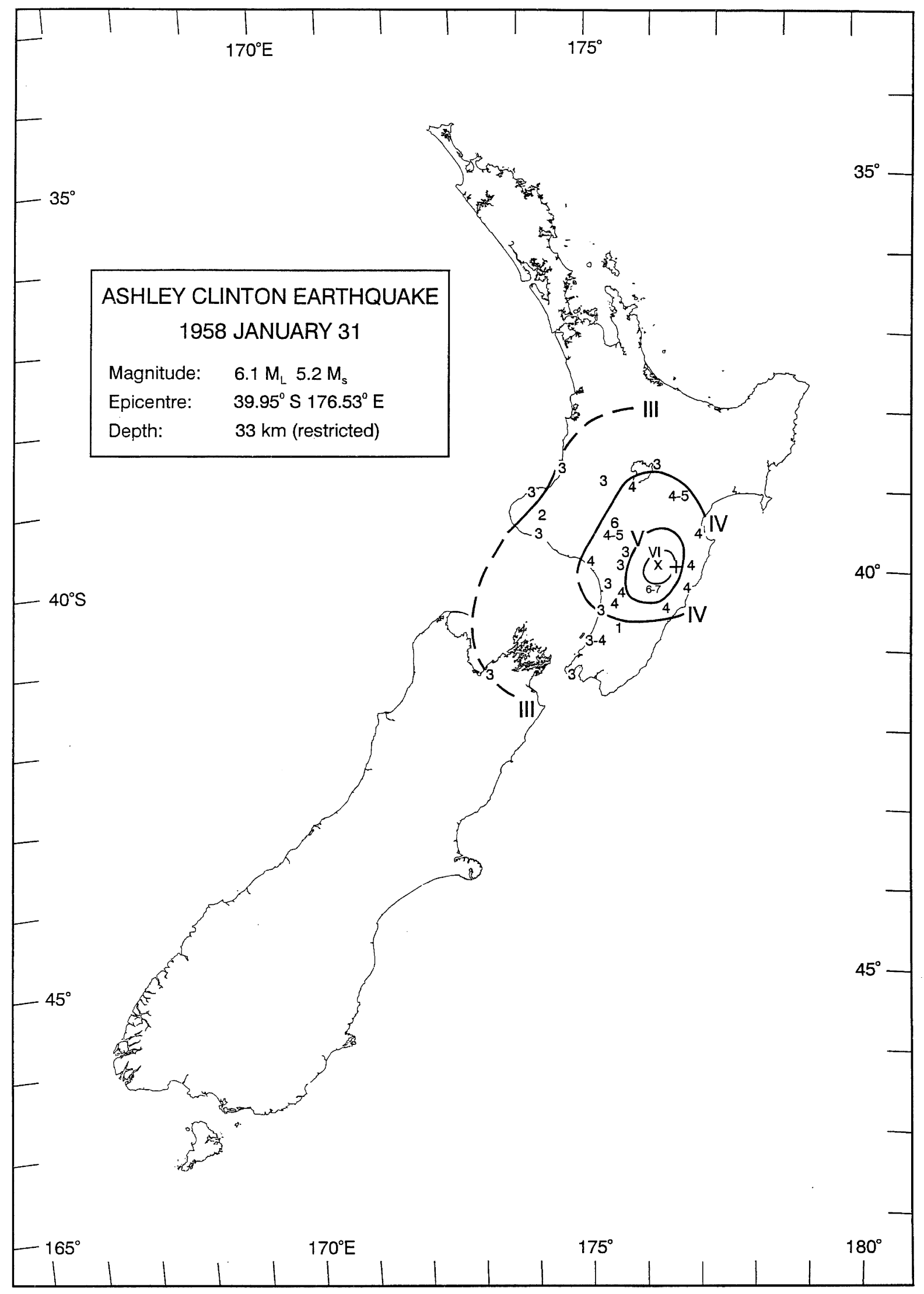

Figure 1: Isoseismals for the magnitude 6.1 Ashley Clinton earthquake (figure from Downes, 1995). 
Care must be taken in interpreting the meaning of coda attenuation (or $\mathrm{Q}_{\mathrm{c}}$ ) factors since the total attenuation of waves in the medium may be due to both scattering and intrinsic absorption which are difficult to separate (Mayeda et al., 1992). Kvamme and Havskov (1989) used spectral ratios of signals of the same event recorded at two different stations and coda decay to show that coda $\mathrm{Q}$ is generally composed of back-scattered S waves. However, some part of the S-wave coda wave train must also contain P-wave coda. Just how much of the S-wave coda is made up of $\mathrm{P}$-wave coda is unclear. Regardless of the coda wave composition, useful observations can be made about the overall effect of $\mathrm{Q}$ on a region since long coda wave trains may be sampling deeper regions in the crust and the overall attenuation is a useful indicator of rock properties.

\section{DATA}

To date we have collected over 20,000 digital seismographic waveforms recorded during the past 10 years from throughout New Zealand. The current national network consists of close to 100 single-component short-period stations. Since 1992 , the network has been slowly upgraded to 3-component seismometers. In addition to the national network, we also used clusters of stations installed near major urban centers near zones of high seismicity or around critical facilities (such as dams). Three key regional networks that we considered in this study are shown in Figure 2. To the north is the Taupo Volcanic zone network, in the southern North Island lies the Wellington network, and in the South Island lies the Clyde network. Each of these regional networks uses single-component seismometers with the exception of Wellington which contains 1 three-component station. To limit ourselves to looking at only upper crustal attenuation properties, we chose to work with only shallow earthquakes less than $15 \mathrm{~km}$ deep.

\section{METHOD}

In this study we used a seismogram stacking technique which is often implemented in inversion schemes (O'Doherty et al. 1997) to highlight regional variations in recorded seismograms. To create the plot in Figure 3, seismograms were gathered for a range of source/receiver distances and bandpass filtered between $1-20 \mathrm{~Hz}$. We then normalized the seismogram by the P-wave amplitude and constructed an envelope along the seismogram (Figure 4). The enveloped seismogram was then added or "stacked" with other seismograms that fit the same source/receiver distance criterion. Noisy or clipped records were down-weighted in the stacks. Finally, we renormalized the final stack to the new P-wave amplitude for plotting. This procedure was adopted for every possible source/receiver distance at $5 \mathrm{~km}$ intervals.

To maximize the use of data for 3 regional networks, we treated each network as a single station and took the seismograms for each event (corresponding to a particular source/receiver distance) and followed the same procedure outlined above. The result is a significant increase in amount of usable data. Whereas a single station might yield 5 seismograms for 5 different earthquakes, a cluster of 5 stations would yield 25 seismograms that can be employed to construct the image in Figure 3.

\section{RESULTS}

The image in Figure 3 illustrates the complexity of seismic raypaths and reveals differences between our crustal model and the actual crust. In the figure we show the estimated arrival times of $P_{n}, P_{g}, S_{n}$, and $S_{g}$ crustal phases which are explained in Figure 4 . While these phases are clearly observed in the stacked seismograms (Figure 3) it is also evident that there is a considerable difference in arrival times of these phases from the model. This stems from the fact that the model was derived from the IASPEI global averaged model. In New Zealand the crustal thickness is often less than the global average of $35 \mathrm{~km}$ resulting in early arrivals as depicted in the plot.

The cloudiness or patchy texture in Figure 3 is due to coda amplitudes for each phase ( $\mathrm{P}$ coda and $\mathrm{S}$ coda) that vary with distance between the source and receiver. Changes are evident between 100 and $200 \mathrm{~km}$ where amplitudes as well as the coda duration appear to increase. These changes are likely due to seismic waves encountering the cross over distance. This distance is that in which reflected and head waves (which travel along an interface) intersect and arrive at the surface simultaneously. Other variations are likely due to azimuthal variations in subsurface geometry (eg. the thickness of the crustal waveguide is not radially symmetric).

Given the clarity of displaying the data in this form, we proceeded to stack seismograms by region to seek regional variations in the character of seismograms. In addition, we chose to subdivide the data even further by selecting seismograms from events whose raypaths were parallel to or perpendicular to the subduction zone. By selectively separating seismograms in this manner we test the notion of the entire South Island having the same subsurface attenuation properties. The resultant plots are shown in Figure 5. The top row of plots in Figure 5 is the combined data (no azimuthal dependence), the middle row are those seismograms with travel paths oriented NE-SW, and the bottom row shows only NW-SE travel paths. The azimuthal variation in coda lengths for the three networks is clear.

The increased coda length of rays coming from NE-SW versus those from NW-SE are evident in all regions except the Taupo volcanic zone which is characterized by high amplitude and long duration signals from all directions. Additionally, the Clyde network shows long coda at $225 \mathrm{~km}$ for both P- and S-waves. Similar features appear, although not as pronounced, in the Wellington data at $200 \mathrm{~km}$

Further details are revealed in these plots if one looks at a single stacked seismogram trace for any given distance. In the Wellington network, for example, the seismogram for events at $102 \mathrm{~km}$ clearly reveals the P-and S-wave arrivals (Figure 6a). A comparison between NE-SW and NW-SE traces shows an average difference of $2 \mathrm{db}$ between the two. If we carry out this exercise for several other traces at different distances the result is surprisingly similar. The difference between NE and NW signals for events from 102, 152,202 , and $202 \mathrm{~km}$ away is about $2 \mathrm{db}$ as shown in Figure $6 \mathrm{~b}$. The interesting feature to these figures is that this difference in coda is almost consistently $2 \mathrm{db}$ out to $300 \mathrm{~km}$. 


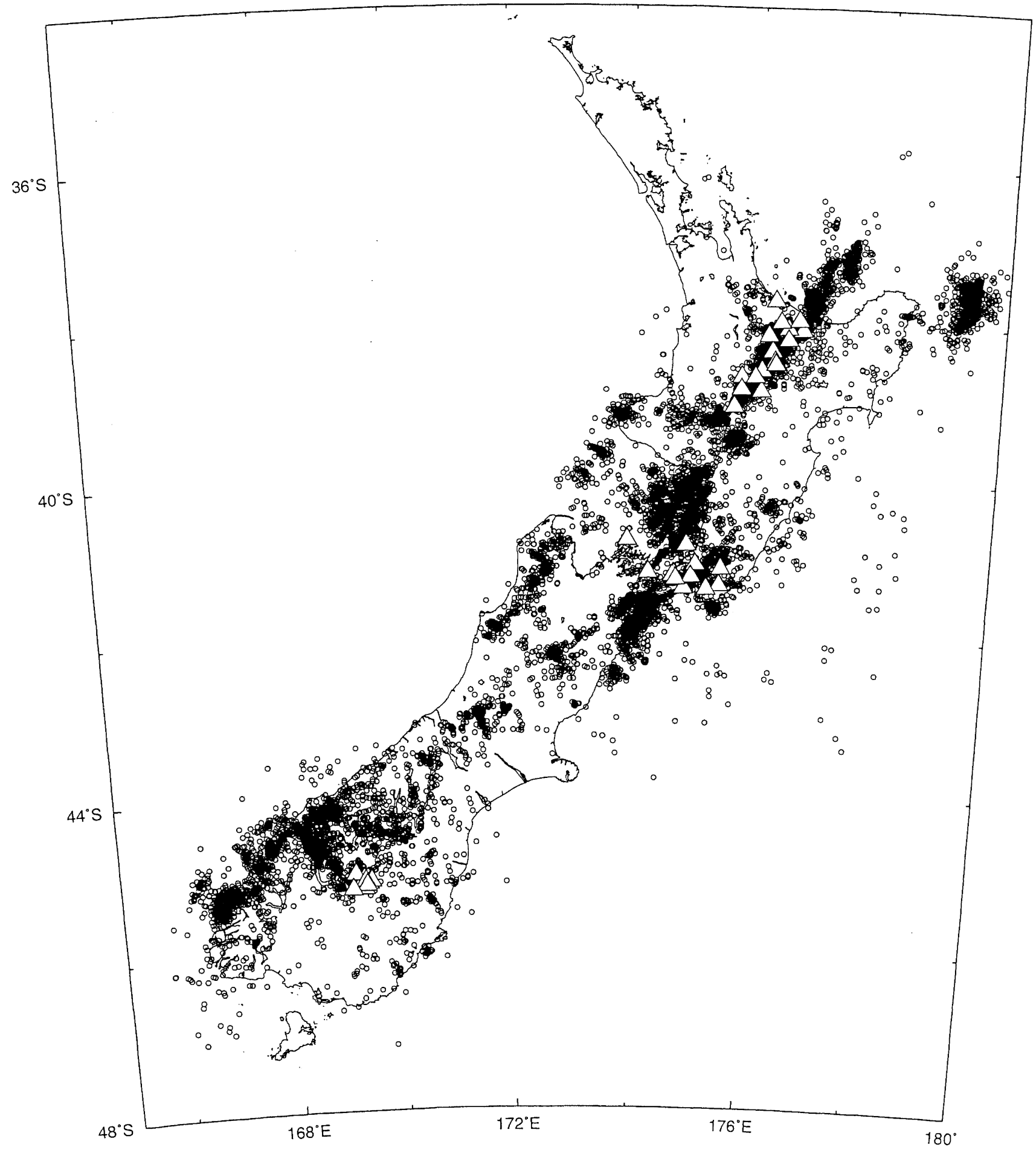

Figure 2: Seismicity of New Zealand from (1988-1997). Seismographic station locations for three regional networks are shown with triangles. For clarity all magnitudes are shown as open circles of the same size. 

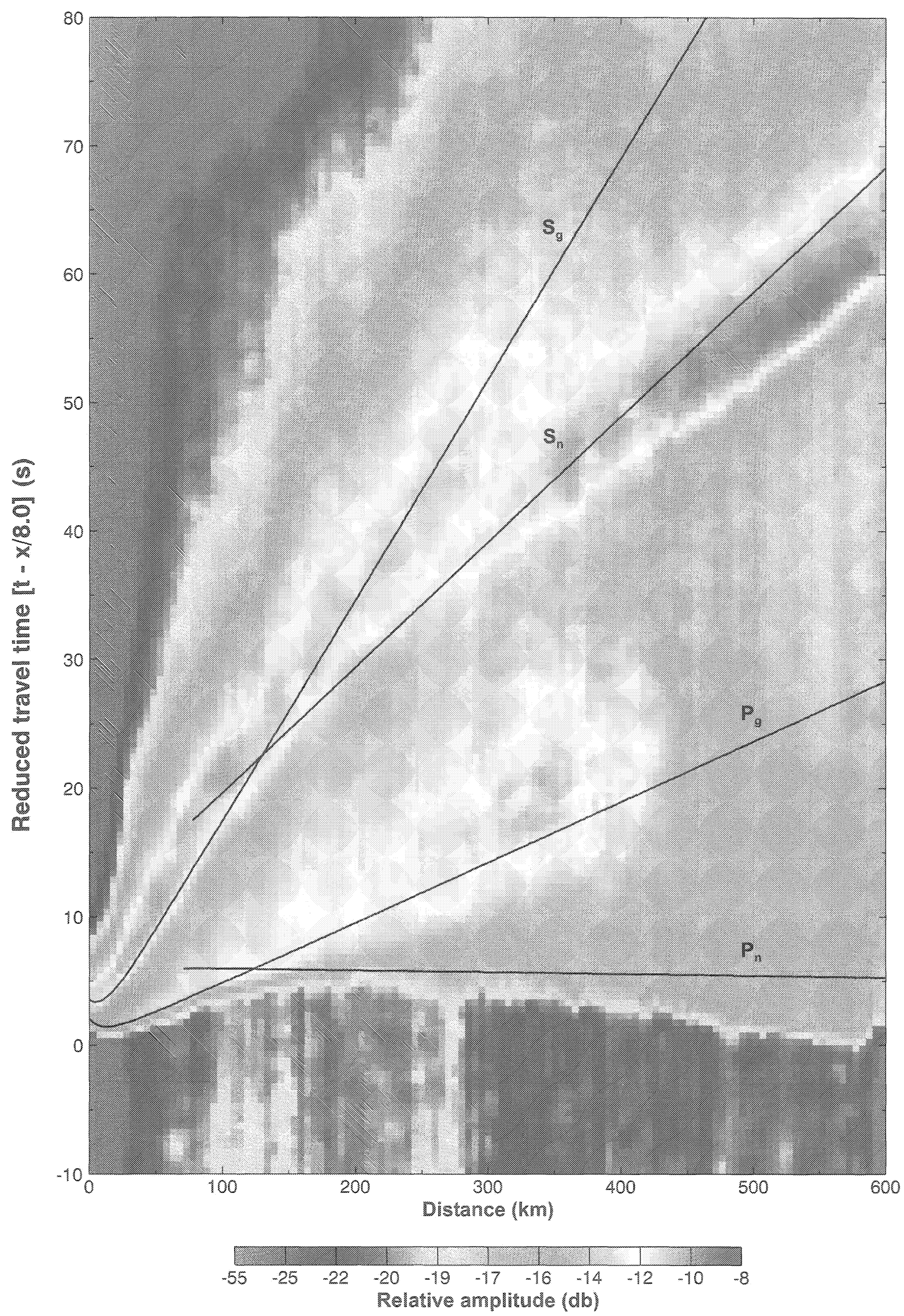

Figure 3: Stack of South Island seismograms for earhquakes less than $15 \mathrm{~km}$ in depth. Superimposed on the image are traces of theoretical arrival times of different $P$ and $S$-waves. The position of these traces is highly dependent upon the cristal velocity model that is used to calculate these arrival wines. 


\section{DISCUSSION AND IMPLICATIONS FOR HAZARD}

The stacking of seismograms is an effective way to use a lot of data that has been accumulated over many years to resolve the structures or physical properties of the earth. We are now at the stage, in terms of technology and the amount of digital data that is currently available, to manipulate the data to reveal these characteristics.

In this paper we used the stacking method to depict regional differences in seismic attenuation in New Zealand. The stacking method has also been directly applied to the tomographic imaging of coda wave attenuation method used by O'Doherty et al. (1997). They claimed that attenuation values derived from coda waves give more stable results and reliable images since they sample space more uniformly than direct $\mathrm{P}$ and $\mathrm{S}$ wave attenuation factors. Here, we show the general characteristics of the waveforms and how they vary throughout New Zealand without proceeding to the full tomographic inversion, which requires a three dimensional velocity model.

Numerous effects may contribute to the azimuthal variation in coda including earthquake mechanisms, topography, and path effects such as near-source geometry and scattering. We believe that earthquake mechanisms and linear sources should not play much of a role here since we have stacked a variety of mechanism orientations thereby averaging out any directivity effects. We suspect that topography might play a more significant role in extending coda duration parallel to the subduction zone since topography is inherently tied to geologic structures and trends. Mountains and valleys are already known to affect radiated energy associated with earthquakes or blasts. A study by Bonner et al. (1996) showed energy from blasts varied azimuthally depending upon whether or not the rays crossed an open pit quarry. On the regional scale that we are considering in this work, perhaps the greatest contribution to azimuthal variation in coda is due to path effects or attenuation. This attenuation may take the form of scattering (as described earlier) or absorption by the medium (intrinsic attenuation).

The results of this work may have important implications for how we calculate the seismic hazard of New Zealand. Since earthquake intensities depend not only on peak values of ground motion (e.g. PGA or peak response) but the entire seismogram, it is likely that we have neglected potentially useful information contained in the full energy packet. The total energy not only includes the peak in the seismogram, but the entire envelope, which includes the duration of shaking. This full packet might be of interest to engineers since the entire time history of the event is preserved. Although the difference in decay between signals perpendicular and parallel to the subduction zone is small, the additional coda length means that the ground should vibrate longer and may thus have a higher capacity for damaging man-made structures. Since we only filter seismograms from $1-20 \mathrm{~Hz}$, more research is needed to focus on the frequency dependency of this approach to identify significant periods in the coda duration.
The azimuthal variation in the attenuation of coda waves may be directly related to the distribution of intensity of shaking. The stacked seismograms show that NE-SW seismograms have longer codas than those to the NW-SE, with the exception of the Taupo Volcanic Zone. This translates into more elliptical isoseismals that trend parallel to the subduction zone. Therefore, rather than accepting radially symmetric attenuation relations which are commonly used in ground motion hazard estimates, there is an additional azimuthal factor that should also be considered. The challenge lies in quantifying and translating the azimuthal variation observed in seismological data to strong motion engineering applications.

\section{CONCLUSIONS}

Seismograms of earthquakes from throughout New Zealand show azimuthal variation in coda waves which verifies prior work with isoseismal distributions. Stacking waveforms in the range of $1-20 \mathrm{~Hz}$ depicts a regional variation in New Zealand attenuation. We believe that most of the directivity effects were averaged out through the stacking of seismograms from earthquakes with different mechanisms and fault orientations. Waveform codas parallel to the subduction zone are generally greater in amplitude and duration than coda perpendicular to the subduction zone. We find that the difference in coda amplitude between NE-SW and NW-SE signals is about $2 \mathrm{db}$.

\section{ACKNOWLEDGMENT}

Funding for this project was generously provided by a grant from the Foundation for Research, Science and Technology, New Zealand. We thank Graeme McVerry , John Zhao and an anonymous reviewer for their review of the manuscript.

\section{REFERENCES}

Aki, K. (1969), Analysis of the seismic coda of local earthquakes as scattered waves, J. Geophys. Res., 74, 615-631.

Aki, K. and Chouet, B., (1975), Origin of coda waves: source, attenuation, and scattering effects, $J$. Geophys. Res,. 80, 3322-3342.

Bonner, J.L., Herrin, E.T., and Goforth, T.T., (1996), Azimuthal variation of $\mathrm{Rg}$ energy from quarry blasts in central Texas, Seismol. Res. Lett., 67, no. 4, 43-56.

Downes, G.L., (1995), Atlas of isoseismal maps of New Zealand earthquakes, Institute of Geological \& Nuclear Sciences Monograph 11, IGNS, Lower Hutt, New Zealand. 
A)

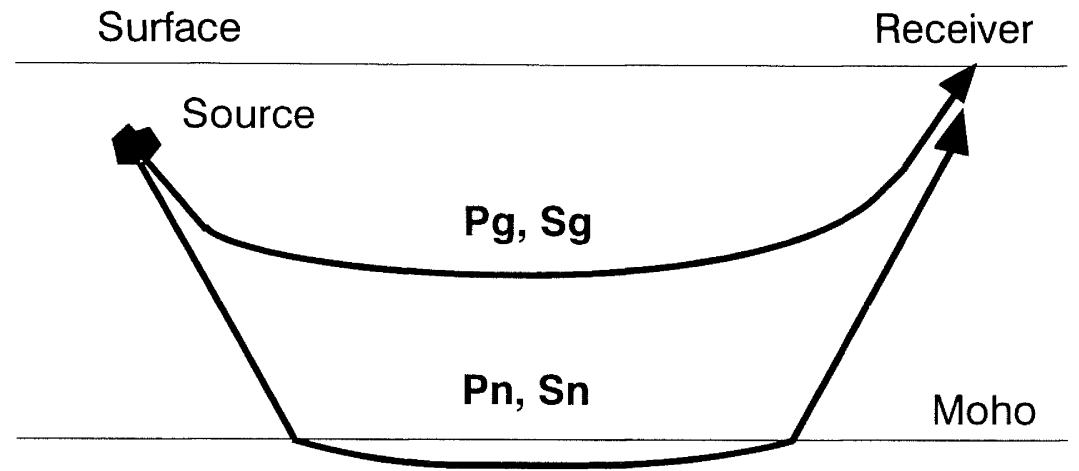

B)

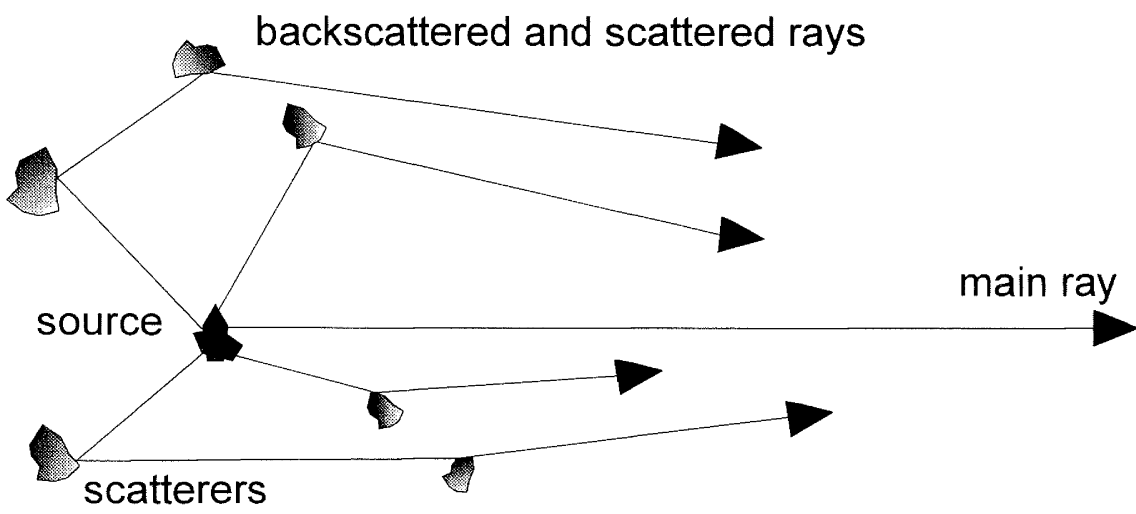

C)

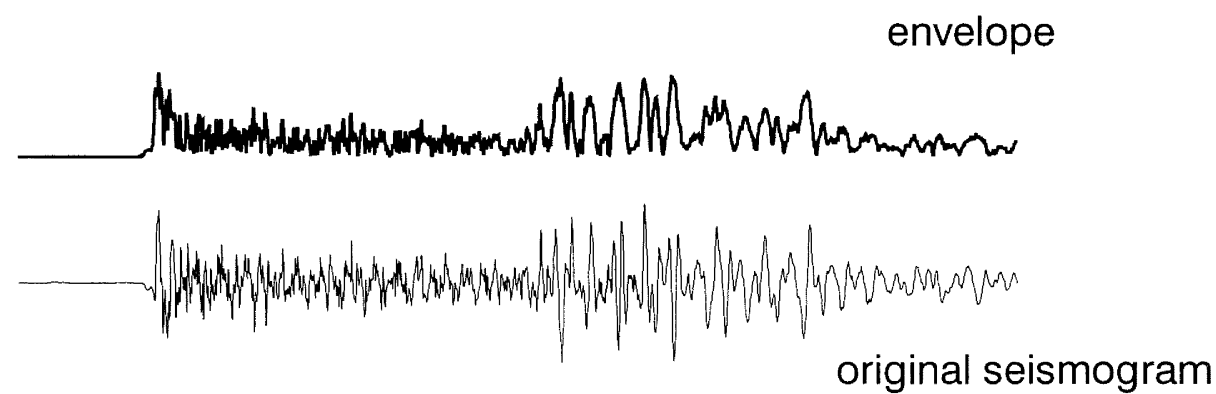

Figure 4: (a) Illustration of the crustal $P$-wave raypaths. The $P_{g}$ phase results from the seismic ray which propagates through the crust in an arcuate ray path from the source to the surface. The $P_{n}$ phase corresponds to the ray which travels at the top of the mantle, in a velocity transition zone just below the Moho interface. Beyond a critical distance in the range of $100-150 \mathrm{~km}$, the $P_{n}$ phase arrives before the more direct $P_{g}$ phase. The same diagram applies to $S$ wave phases. (b) Cartoon depicting the coda backscattering along the main ray path which contributes to long coda signals. (c) Comparison of an original seismogram with the constructed envelope that is used in the stacking process. Note the positive nature of the envelope which prevents negative and positive amplitudes from canceling out in the stacking process. The long wave trains which appear after the main phases $(P$ - and $S$-) are due to scattering effects. 

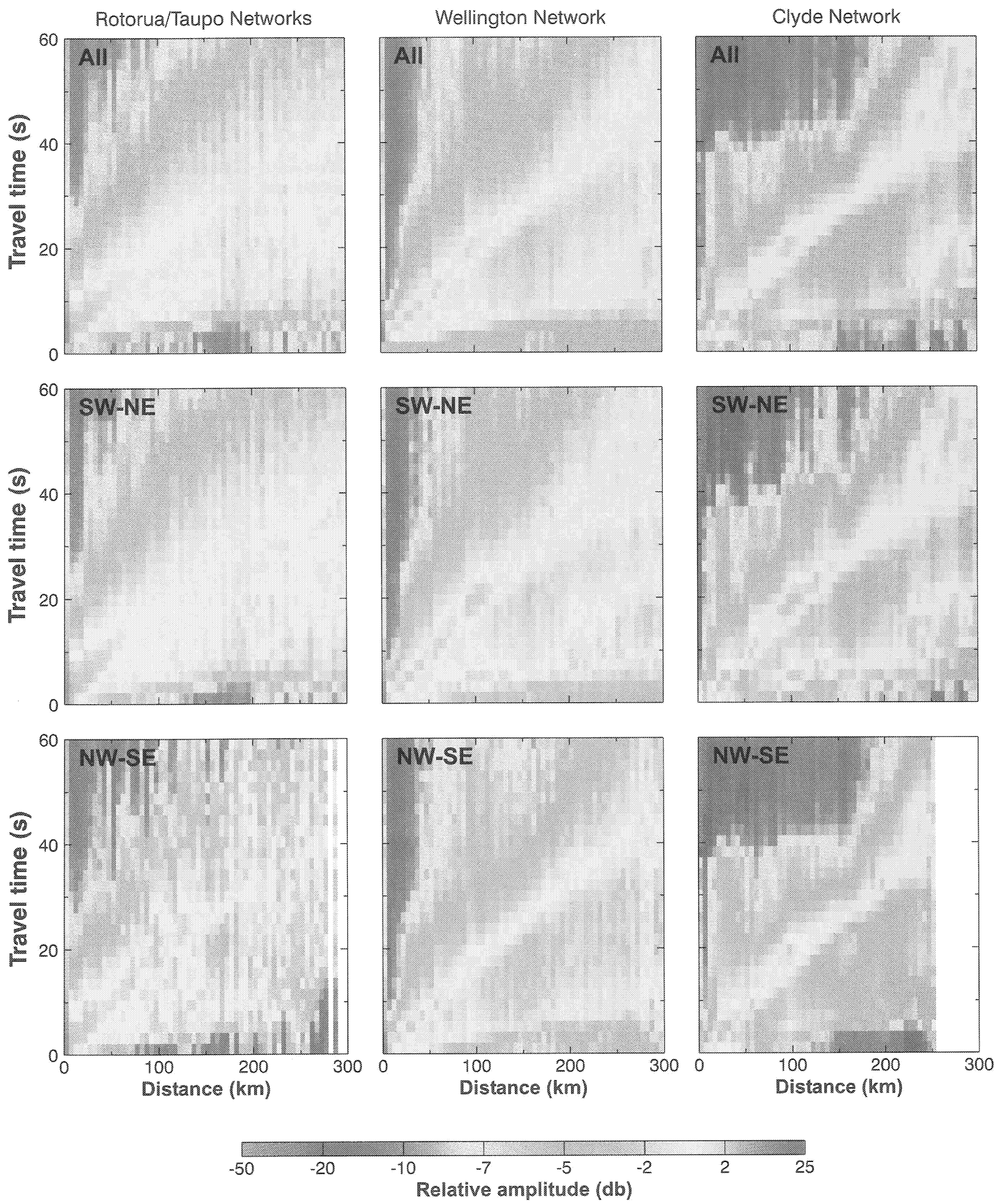

Figure 5: Stack of regional network seismograms. The top row is for all azimuths, the middle row is NEmW azimuths, and the bottom row is NW.SE azimuths. 
Dowrick, D.J., (1991), A revision of attenuation relationships for modified Mercalli intensity in New Zealand earthquakes, Bull. NZ Nat. Soc. for Earthquake Engineering, 24, no. 3, 210-224.

Kozuch, M.J., Smith, E. and Vere-Jones, D., (1996), Modeling New Zealand isoseisms with site-specific attenuation (abstract), Eos, Trans. Am. Geophys. Union, 77, no. 46, F510.

Kozuch, M.J. and Chadwick, M., (1997), Isoseismal distributions for New Zealand earthquakes verified with azimuthal and regional variations in coda waves (abstract), Eos, Trans. Am. Geophys. Union, 78, no. 46, F434.

Kvamme, L.B. and Havskov, J., (1989), Q in southern Norway, Bull. Seismol. Soc. Amer., 79, 1575-1588.

Mayeda, K., Koyanagi, S., Hoshiba, M., Aki, K. and Zeng, Y., (1992), A comparative study of scattering, intrinsic, and coda Q-1 for Hawaii, Long Valley, and Central California between 1.5 and $15.0 \mathrm{~Hz}, J$. Geophys. Res.,97, 6643-6659.

O'Doherty, K.B., Bean, C.J. and McCloskey, J., (1997), Coda wave imaging of the Long Valley caldera using a spatial stacking technique, Geophys. Res. Lett., 24, $1547-1550$.
Pulli, J.J., (1984), Attenuation of coda waves in New England, Bull. Seismol. Soc. Amer., 74, 1149-1166.

Sato, H., (1977), Energy propagation including scattering effects: single isotropic scattering, J. Phys. Earth, 25, 27-41.

Smith, W.D., (1978), Spatial distribution of felt intensities for New Zealand earthquakes, NZ J. Geol. Geophys., 21, 293-311.

Smith, W.D., (1995), A development in the modelling of farfield intensities for New Zealand earthquakes, Bull. N.Z. Nat. Soc. for Earthquake Engineering, 28, 196217

Vere-Jones, D., Pang, J. and Smith, E.G., (1995), Statistical modelling of felt intensity data for three New Zealand earthquakes, Report to the NZ Earthquake Commission. Institute of Stats. and Operations Res., Victoria University of Wellington, 41.

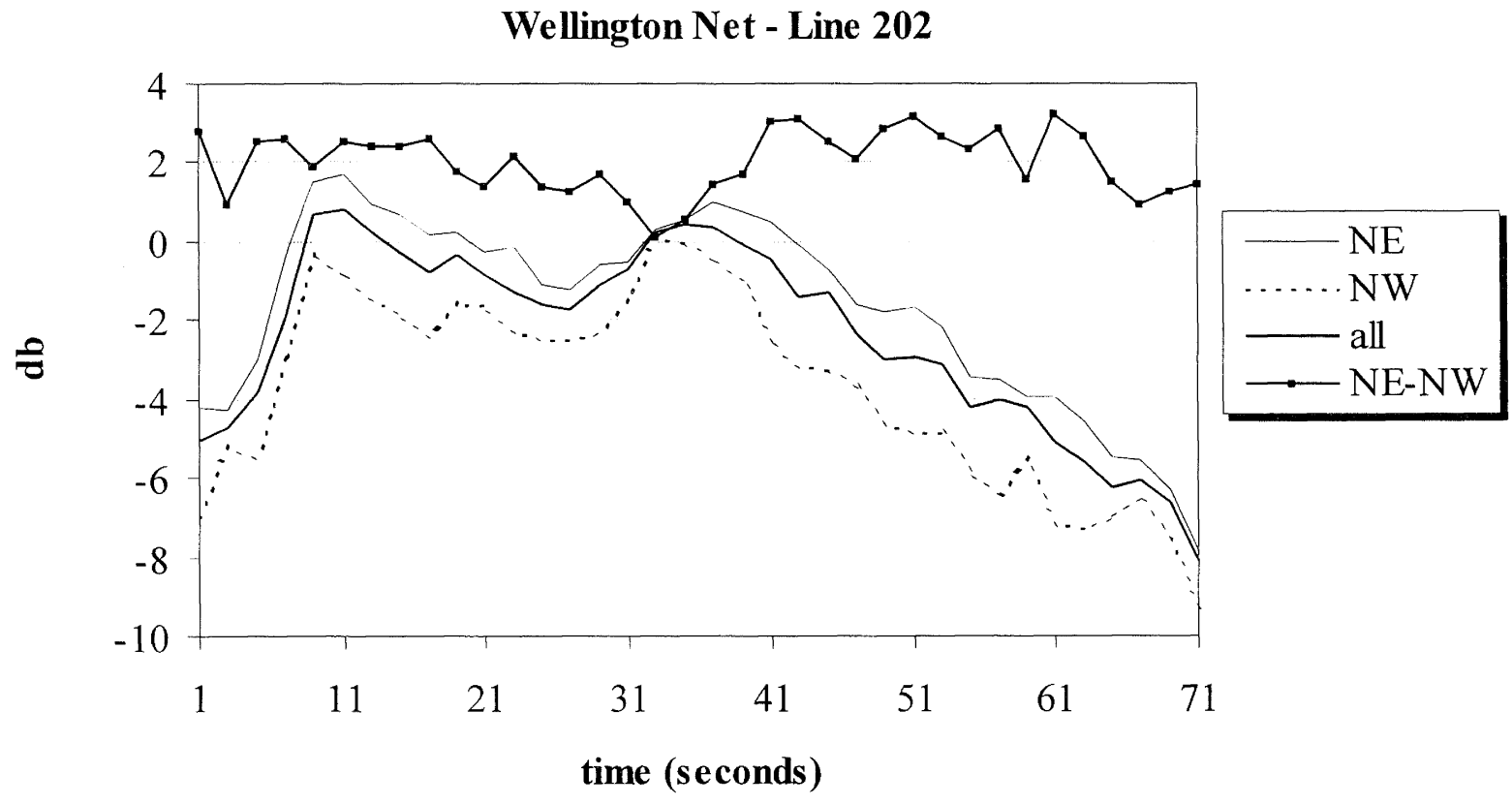

Figure 6a: Seismogram traces taken from the Wellington network at distance of $102 \mathrm{~km}$ for the NE-SW seismograms (ne), the NW-SE seismograms (nw), and all azimuths (all). The $P$-wave is visible at 9 seconds while the $S$-wave arrives at 37 seconds. The uppermost trace shows the difference between the NE-SW traces and NW-SE seismograms $(N E-N W)$. Each trace is composed of several stacked seismograms, which have been filtered and normalized. 


\section{Wellington Net \\ difference between NW and NE stacked waveforms}

at four different distances

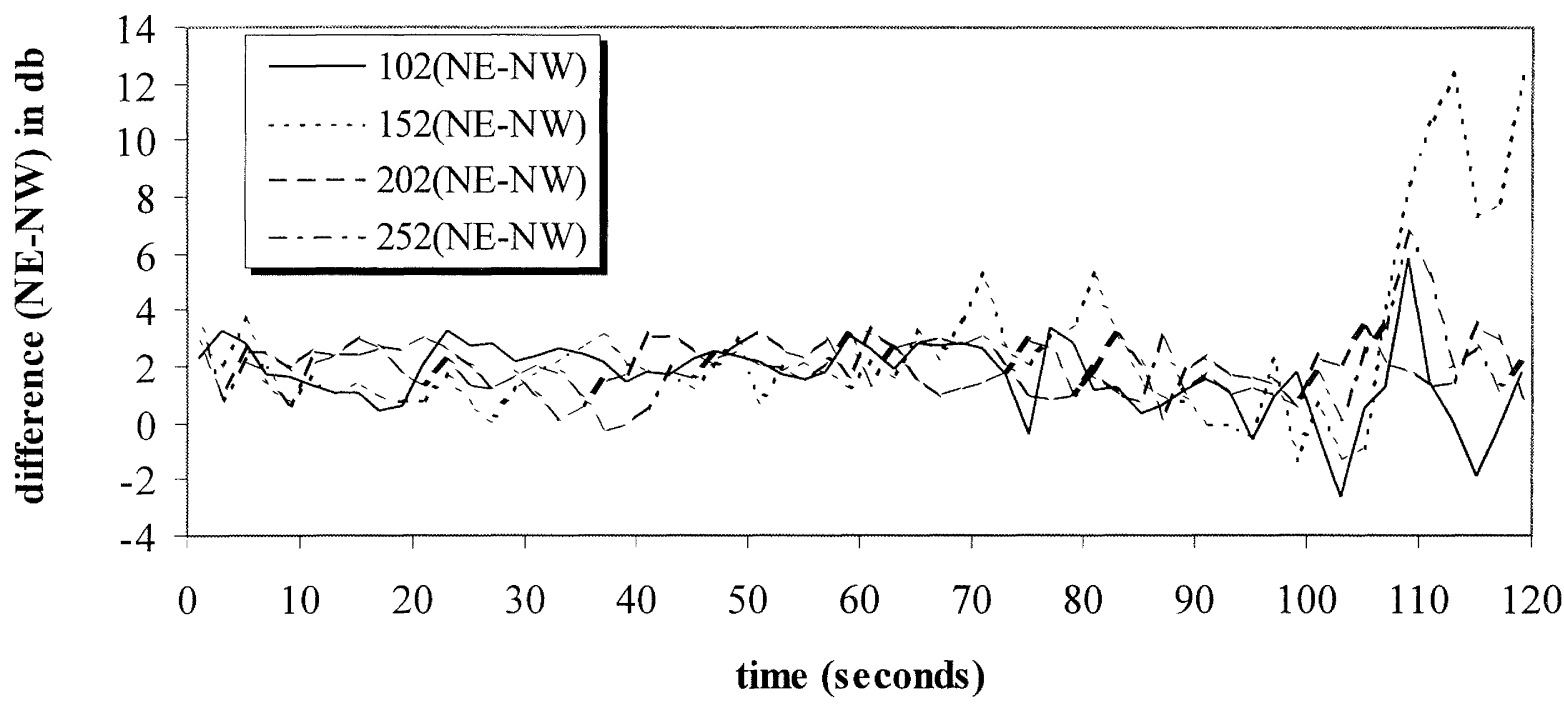

Figure 6b: The difference between NE-SW seismograms and NW-SE seismograms taken at distances of 102, 152, 202, and $252 \mathrm{~km}$ for the Wellington network. Each of these distance designations is actually a range of distances (e.g. 102 is composed of seismograms from 100-105 $\mathrm{km}$ between the source and receiver). 